\title{
TNF- $\alpha$ is not the cause of fatty liver disease in obese diabetic mice
}

To the editor-The article by Lin et al. ${ }^{1}$ in the September 2000 issue of Nature Medicine proposed that metformin treatment reverses fatty liver disease (FLD) in obese, leptin-deficient (ob/ob) mice by decreasing hepatic TNF- $\alpha$ mRNA expression. In that study, metformin reversed hepatomegaly, lowered elevated serum transaminase levels, and decreased TNF$\alpha$, uncoupling protein 2 (UCP2) mRNA and fatty acid synthase protein in the liver in ob/ob mice. Because earlier studies have shown that TNF- $\alpha$ is elevated in ob/ob mice, stimulates hepatic fatty acid synthesis and induces UCP2 expression in the liver, the authors postulated that TNF- $\alpha$ and TNF-inducible factors that promote hepatic lipid accumulation contribute to FLD in obese mice.

While the hypothesis that increased hepatic TNF- $\alpha$ expression caused FLD is attractive, it is also conceivable that the increase in hepatic TNF- $\alpha$ expression is a consequence of lipid accumulation rather than the cause. One approach to obtain definitive evidence for or against the role of TNF- $\alpha$ as a mechanism of FLD is to use knockout mice. Previous studies have shown that ob/ob mice that lack both TNF receptors (ob/ob-p55/p75-null) and therefore lack functional TNF- $\alpha$ activity, have improved insulin sensitivity ${ }^{2}$, but no change in body weight or fat mass per lean body mass ${ }^{3}$. We have reported that ob/ob-p55/p75-null mice have twice as much UCP2 expression in the liver and adipose tissue compared with ob/ob mice $^{4}$ suggesting that en dogenous TNF$\alpha$ has an inhibitory effect on UCP2 and therefore a lack of TNF- $\alpha$ further upregulates UCP2 expression in obese mice. Since these data were in contrast to the proposed hypothesis of Lin et al. ${ }^{1}$ that TNF- $\alpha$ may be the cause of FLD and subsequent susceptibility to liver toxicity in ob/ob mice, we have measured hepatic lipid content and serum transaminase levels in control, ob/ob, p55/p75-null and ob/ob-p55/p75-null mice.

We show that hepatic lipid content is similar in ob/ob and ob/ob-p55/p75null mice (Table 1). Moreover, serum transaminase levels are also not significantly different between ob/ob mice with or without TNF receptors. Thus, these data demonstrate that lack of TNF$\alpha$ action neither reverses hepatic lipid accumulation nor lowers el evated serum transaminase levels in obese mice, indicating that TNF- $\alpha$ is not the mechanism of FLD in obese diabetic mice. Our data indicate that FLD occurs in ob/ob mice, even in the absence of TNF action and TNF-induced insulin resistance.

\section{RiazA. Memon, Carl Grunfeld \&}

Kenneth R. Feingold

Department of Medicine,

University of California, San Francisco, USA

M etabolism Section,

Veterans Affairs M edical Center,

San Francisco, California, USA

Email: rmemon@itsa.ucsf.edu

Table 1 Total hepatic lipid content and serum transaminase levels in ob/ob and ob/ob-p55/p75-null mice.

$\begin{array}{lccc}\text { Experimental Group } & \begin{array}{c}\text { Hepatic Lipid } \\ \text { Content } \\ \text { (mg/g wet weight) }\end{array} & \begin{array}{c}\text { Serum Alanine } \\ \text { Aminotranferase } \\ (\mathrm{U} / \mathrm{L})\end{array} & \begin{array}{c}\text { Serum Aspartate } \\ \text { Aminotransferase } \\ (\mathrm{U} / \mathrm{L})\end{array} \\ \text { Control mice } & 29.2 \pm 1.2 & 13.6 \pm 0.6 & 19.9 \pm 3.4 \\ \text { ob/ob mice } & 59.1 \pm 1.4 & 46.4 \pm 4.2 & 35.5 \pm 6.1 \\ \text { P vs. control } & <0.001 & <0.001 & \mathrm{NS} \\ \text { p55/p75-null mice } & 34.1 \pm 1.3 & 14.8 \pm 4.3 & 19.7 \pm 3.6 \\ \text { P vs. control } & <0.05 & \mathrm{NS} & \mathrm{NS} \\ \text { ob/ob-p55/p75-null mice } & 57.6 \pm 3.1 & 47.6 \pm 12.8 & 44.1 \pm 11.9 \\ \text { P vs. p55/p75-null mice } & <0.001 & <0.05 & \mathrm{NS}\end{array}$

Age matched control, ob/ob, p55/p75-null and ob/ob-p55/p75-null mice (8-wk-old male mice, $n=5$ in each group) were maintained on chow and water ad libitum. After 1 wk mice were killed; total hepatic lipid content and serum transaminase levels measured by standard methods. Data are presented as mean $\pm \mathrm{s}$.e.m; analysis of variance was used to determine statistical significance. All mice gifts of G.S. Hotamisligil (Harvard University, Boston, Massachussetts\}.

Diehl replies-Memon and colleagues re port that hepatic lipids and serum aminotransferases are similar in ob/ob wild-type mice and ob/ob mice that are genetically deficient in both TNF receptors, apparently refuting our hypothesis that increases in hepatic TNF- $\alpha$ contribute to the pathogenesis of FLD. Recently, they also noted increased UCP2 mRNA levels in the livers of TNF- $\alpha$ receptor-deficient ob/ob mice ${ }^{4}$, casting further doubt on our suggestion that TNF-related induction UCP-2 compromises hepatocyte viability in fatty livers. It is important, however, to emphasize that several issues remain un resolved.

Serum aminotransferase values were normal in both strains of ob/ob mice that Memon and colleagues studied. We find, however, that serum aspartate aminotransferase (AST) and alanine aminotransfarese (ALT) values in adult male ob/ob mice are significantly higher than in age- and gender-matched litter mates $^{1,6}$. These differences might be important because FLD begins with fatty liver (steatosis) and progresses to fatty liver with inflammation and liver cell injury (steatohepatitis). The release of liverderived aminotransferases into the serum occurs predominately during steatohepatitis ${ }^{7}$. Thus, the ob/ob mice that we studied had steatohepatitis, while Memon's ob/ob mice were probably at the earlier, steatotic stage. Another group has reported that TNFreceptor-deficient mice are completely protected from steatohepatitis induced by alcohol, demonstrating the importance of TNF- $\alpha$ at the inflammatory stage of fatty liver disease ${ }^{8}$.

Moreover, alcohol- or obesity-induced fatty livers are unusually vulnerable to TNF- $\alpha$-mediated injury ${ }^{6,8}$. This vulnerability results partly from hepatocyte mitochondrial adaptations to chronic oxidative stress ${ }^{9,10}$. We proposed that activation of mitochondrial uncoupling proteins might be involved in this process after noting that UCP-2 mRNA, protein and activity are increased in ob/ob fatty hepatocytes but repressed in ob/ob macrophages $^{11}$. This pattern of UCP-2 expression differs from normal livers, where UCP-2 is localized predominantely in macrophages and barely expressed in hepatocytes $^{12}$. Because TNF- $\alpha$ upregulates 